\title{
International Session 1
}

\section{Korean experience in septorhinoplasty}

\author{
Hun-Jong Dhong \\ Department of Otorhinolaryngology - Head Neck Surgery, Samsung Medical Center, \\ Sungkyunkwan University School of Medicine
}

The Korean rhinologic society has eight study groups, which are allergy and immunology study group, sinusitis and polyp, olfaction and gustation, sleep and sleep physiology, airway mucosa physiology, facial plastic surgery, sinonasal tumor, tissue engineering and regenerative medicine. The number of active member of facial plastic surgery is about 120 , which was 25 when the facial plastic surgery study group developed in 2004. Rhinoplasty performed by rhinologist has become more popular and more socially accepted over the last decade. Corrective rhinoplasty to improve nasal function and aesthetics was the most commonly performed procedure when the study group was organized. However, cosmetic rhinoplasty has become increased, and furthermore young doctors have expanded our interest and performance beyond rhinoplasty. These days, double eyelid surgery, facial rejuvenation, otoplasty, and face lift are getting common topics in symposium, and also performed in practice by ENT doctors. In 2010, we, rhinologist, developed more professional society for this field, of which name is the Korean Academy of Facial Plastic and Reconstructive Surgery (KAFPRS). Facial plastic surgery study group still exist in KRS.

Most Koreans have platyrrhine chracteristics with a low dorsum and inadequate projection. The concepts of beauty nurtured by Asians may not conform to the western ideal of beauty. Beauty is an ill-defined concept that is recognized cross-culturally. It is difficult to quantify across different ethnic groups. To achieve high levels of patient satisfaction after rhinoplasty, the surgeon should understand appropriate aesthetic norm. Because of the enormous cultural influence of the western media, some patients prefer Caucasian nose. In the initial evaluation, discussion should begin by focusing on the patient's realistic expectation with a frank discussion of the limitations of the procedures based on the patient's anatomy. The anatomy and dimensions of the Korean nose have been found to be quite different from the Caucasian nose in many aspects. Korean nose is smaller relative to the face than is the white nose. They tend to have weak lower lateral cartilages with thick sebaceous skin over the nasal tip, leading to a blunting of the nasal contours. It has been observed that the Asian nose usually has a broad alar base and short, thick nasal bone.

Because of these characteristics, commonly performed procedures are augmentation rhinoplasty and tip projection techniques in these days. Patients want to get more projected and well demarcated nose. Applying surgical principles for whites to Koreans often produces unexpected and sometimes even disastrous results. General goals for rhinoplasty should include the improvement of the nasal respiratory function and satisfied aesthetic improvement with preservation of the nasal support structures. Asian rhinoplasty should be tailored best to suit the Asian face with a size and shape that falls within the range of naturally occurring noses in this population. From an aesthetic point of view, the Asian nose tolerates less change than other ethnic noses. 